EPJ Web of Conferences 38, 08001 (2012)

DOI: $10.1051 /$ epjconf/20123808001

(c) Owned by the authors, published by EDP Sciences, 2012

\title{
Perspectives for photofission studies with highly brilliant, monochromatic $\gamma$-ray beams
}

\author{
P.G. Thirolf ${ }^{1, \text { a }}$, L. Csige ${ }^{1}$, D. Habs ${ }^{1,2}$, M. Günther ${ }^{2}$, M. Jentschel ${ }^{3}$, A. Krasznahorkay ${ }^{4}$, D. Filipescu ${ }^{5}$, T. Glodariu ${ }^{5}$, \\ L. Stroe ${ }^{5}$, O. Tesileanu ${ }^{5}$, H. Karwowski ${ }^{6}$, and G. Rich ${ }^{6}$ \\ 1 Ludwig-Maximilians-Universität München, Garching, Germany \\ 2 Max-Planck-Institut f. Quantenoptik, Garching, Germany \\ 3 Institut Laue-Langevin, Grenoble, France \\ 4 Institute of Nuclear Research of the Hungarian Academy of Science (ATOMKI), Debrecen, Hungary \\ 5 Horia Hulubei National Institute of Physics and Nuclear Engineering, IFIN-HH, Bucharest, Romania \\ 6 Triangle Universities Nuclear Laboratory, Durham and Univ. of North Carolina, Chapel Hill, USA
}

\begin{abstract}
New research facilities like MEGa-Ray (Livermore) or ELI-NP (Bucharest) will provide within the next years (2013-2016) photon beams of unprecedented quality with respect to both photon intensity (total flux $\left.10^{13} \mathrm{\gamma} / \mathrm{s}\right)$ and spectral intensity $\left(\sim\left(10^{4}-10^{6}\right) / \mathrm{eVs}\right)$, thus exceeding the performance of existing facilities by several orders of magnitude. This tremendous progress will be enabled by Compton-backscattering of an intense laser off a high-quality electron beam, in conjunction with novel refractive bremsstrahlung beams focusing $\gamma$ optics and efficient monochromatization techniques. We envisage to employ these $\gamma$ beams for photofission studies on extremely deformed nuclear states of actinides, investigating their multiple-humped potential energy landscape in a highly selective way. Transmission resonances in the prompt fission cross section from the (superdeformed) second and (hyperdeformed) third potential minimum will be studied, where the fission decay channel can be expressed as a tunnelling process of these gateway states through the multiple-humped fission barrier.
\end{abstract}

\section{Novel brilliant $\gamma$ beams for photonuclear science}

Photonuclear physics is a well-established field of research since decades [1]. Mostly bremsstrahlung has been used to excite nuclei or to induce fission in the actinides [2]. A significant improvement in narrowing the energy resolution of photon beams from typically $200-300 \mathrm{keV}$ at bremsstrahlung facilities to about $15-25 \mathrm{keV}$ was achieved by introducing the tagged-photon technique [3]. In recent years increasingly also Compton-backscattering of (laser-) photons off an energetic electron beam was used to generate $\gamma$ beams according to the scheme illustrated by figure 1 . Here the Doppler-upshift experienced by the initial pho-

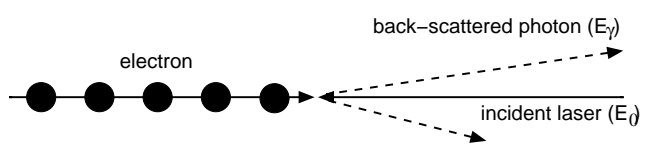

Fig. 1. Sketch of the mechanism to produce $\mathrm{MeV}$ photon beams $\left(\mathrm{E}_{\gamma}\right)$ by exploiting the Doppler-upshift experienced by a laser photon (energy $\mathrm{E}_{0}$ ) which is Compton-backscattered off a relativistic electron.

ton with energy $E_{0}$ is exploited to generate photon beams with high energies $\mathrm{E}_{\gamma}$ via Compton-backscattering from a counter-propagating fast electron beam, characterized by its relativistic factor $\gamma$ according to

\footnotetext{
${ }^{a}$ e-mail: Peter.Thirolf@lmu.de
}

$$
E_{\gamma}=4 \gamma^{2} \cdot \frac{1}{1+(\theta \gamma)^{2}+\frac{4 \gamma E_{0}}{m c^{2}}} \cdot E_{0} \approx 4 \gamma^{2} E_{0}
$$

The simplified scaling of the backscattered photon energy with $4 \gamma^{2}$ holds for exactly backscattered photons and takes into account that the recoil term $4 \gamma E_{0} / m c^{2}$ is negligible for initial photon energies of a few eV. In realistic experiments, the angular spread after Compton scattering, described within the Klein-Nishina formula by the angle $\theta$ between the incident laser direction and the direction of the scattered $\gamma$ beam, will be reduced by appropriate collimation of the back-scattered photons.

Presently the world-leading $\gamma$-beam facility $\mathrm{HI} \gamma \mathrm{S}$ (High Intensity Gamma-Ray Source) is operated at Duke University in the US [4]. A free-electron laser (FEL) operated in a $1.2-\mathrm{GeV}$ electron storage ring is used to produce photons (from IR to VUV) that subsequently are Comptonbackscattered off the relativistic electron beam. Currently, $\mathrm{HI} \gamma \mathrm{S}$ can produce $\gamma$ rays between 2 and $100 \mathrm{MeV}$ with linear and circular polarizations. Total $\gamma$-ray intensities can reach over $10^{9}$ photons/second at some energies, reduced by about an order of magnitude when collimating the beam to an energy resolution of ca. 5\% (FWHM). Recently an upgrade proposal has been submitted ('HI $\gamma \mathrm{S} 2$ '), aiming at a drastic increase of the $\gamma$-beam brilliance and spectral intensity (see Tab. 1) by replacing the present FEL by an intense laser beam.

At the Lawrence Livermore National Laboratory, the 'MEGa-ray' project is pursued, aiming at the production of monoenergetic multi-MeV $\gamma$ beams from laser-Compton 
backscattering [5]. Short pulses from an intense laser collide head-on with short bunches of electrons, accelerated in a compact linear accelerator. The present MEGa-ray project represents already the third generation of Compton backscattering $\gamma$ sources realized by the Livermore group. Starting from the PLEIADES project [6], designed for generating $\mathrm{X}$-ray radiation of $70 \mathrm{keV}$, the photon energy was increased by about an order of magnitude in the second generation, the T-REX project [7]. In MEGa-ray, photon energies up to about $4 \mathrm{MeV}$ are envisaged in a highly brilliant, monochromatic $\gamma$ beam. In order to keep the electron accelerator compact, X-band technology at an acceleration frequency of ca. $12 \mathrm{GHz}$ will be employed. A total photon flux of $10^{13} \mathrm{\gamma} / \mathrm{s}$ and a so far unprecedented energy bandwidth of $\Delta \mathrm{E} / \mathrm{E} \leq 10^{-3}$ are targeted.

While in the past in Europe only bremsstrahlung was employed for photonuclear physics (e.g. the Dynamitron in Stuttgart [8] and the bremsstrahlung facility at the SDALINAC in Darmstadt, recently upgraded to operate also with tagged photons [3]), presently a new large-scale research facility is close to its start of construction. The 'Extreme Light Infrastructure' (ELI) initiative [9] will be built in a pan-European effort by a joint European consortium of 17 nations as a 'distributed facility' consisting of three individual high-power laser facilities that will be consolidated under the joint ELI project. The prime objective is to build a unified infrastructure based on (initially) three mutually supporting pillars. One pillar will be located in Prague (Czech Republic), focusing on providing a novel generation of secondary sources from high energy laser beams for interdisciplinary applications in physics, medicine, biology, and material sciences. The second pillar, concentrating on the physics of ultrashort optical pulses on the attosecond scale, is planned for location in Szeged (Hungary). Finally, the third pillar will be built in Magurele, close to Bucharest (Romania) and will be dedicated to (photo-)nuclear physics [9], therefore termed ELI-Nuclear Physics (ELI-NP). One of the central features of ELI-NP will be the $\gamma$-beam facility, aiming at providing highly-brilliant photon beams up to $19 \mathrm{MeV}$ with an energy bandwidth of better than $10^{-3}$ and a total photon flux of about $10^{13} \mathrm{\gamma} / \mathrm{s}$. An option for its realization could be to scale the technology developed for MEGa-Ray to the envisaged $750 \mathrm{MeV}$ electron linac. ELI-NP includes, besides the $\gamma$ beam, also two 10 PW lasers, where also e.g. laser acceleration of heavy elements as joint experiments between the $\gamma$ beam and the laser beam are under consideration.

ELI-NP, representing a project volume of about 300 MEUR financed by European structural funds, will commence construction still in 2012 and is aiming at starting operation at the end of 2017.

Tab. 1 shows a comparison of the performance of the above discussed $\gamma$-beam facilities.

Moreover, it should be noted that presently a novel development is ongoing with the potential to drastically further improve the performance of $\gamma$-beam facilities. In contrast to textbook knowledge, recent experiments revealed the existence of a much larger index of refraction for energetic (MeV-class) photons in silicon than conventionally expected [10]. This finding is explained by the previously underestimated influence of Delbrück scattering, i.e. the scattering of $\gamma$ rays by the extremely strong electric field of an atomic nucleus. If further experiments succeed to con-
Table 1. Comparison of performance properties of existing and upcoming $\gamma$-beam facilities.

\begin{tabular}{l|llll}
\hline & $\mathrm{HI} \gamma \mathrm{S}$ & $\mathrm{HI} \gamma \mathrm{S} 2$ & MEGa-Ray & ELI-NP \\
\hline $\mathrm{E}_{\gamma}[\mathrm{MeV}]$ & $2-100$ & $2-100$ & $<8$ & $<19$ \\
total flux $[\gamma / \mathrm{s}]$ & $\sim 10^{9}$ & $5 \cdot 10^{12}$ & $10^{13}$ & $10^{13}$ \\
$\begin{array}{l}\Delta \mathrm{E} / \mathrm{E} \\
\text { spectral density }\end{array}$ & $2-5 \cdot 10^{-2}$ & $\leq 10^{-3}$ & $\leq 10^{-3}$ & $\leq 10^{-3}$ \\
{$[\gamma / \mathrm{eVs}]$} & & $10^{4}-10^{6}$ & $10^{4}-10^{6}$ & $10^{4}-10^{6}$ \\
\hline
\end{tabular}

firm the expected strong Z-dependence of this effect, this would enable the development of focusing refractive optical elements for $\mathrm{MeV} \gamma$ beams, with the perspective to significantly improve the properties of $\gamma$ beams way beyond the parameters listed in Tab. 1, e.g. by the capability to parallelize the inherently divergent Compton-backscattered photon beams prior to their efficient monochromatization in a double-crystal monochromator.

\section{The multiple-humped potential barrier in the actinides}

Exploring the potential energy landscape of actinide nuclei allows to gain insight into a variety of highly relevant issues in current nuclear physics research. Improving our knowledge on fission barriers of heavy nuclei allows for quantitative tests of, e.g., macroscopic-microscopic nuclear models, which can lead to refined predictions of production cross sections for very heavy or super-heavy elements or to an improved input for astrophysical network calculations.

Spectroscopy of nuclear configurations at large deformations is of particular interest in actinide nuclei: in contrast to lower-mass regions, superdeformed configurations can be accessed in actinide isotopes already at low angular momenta, giving rise to a clear separation between vibrational and rotational spectroscopic features. Studying single-particle Nilsson orbitals at large deformations allows to probe less deformed down-sloping orbitals from higher-lying shells. In addition to spectroscopic information, measuring angular, mass and charge distributions of fission fragments originating from strongly deformed nuclear states of the fissioning system enables to study dynamical aspects of the fission process.

Throughout the last 15 years, our picture of the multiplehumped actinide fission barrier has matured significantly, mostly owing to improved detection technologies $[11,12]$. While the (superdeformed) second potential minimum with an axis ratio of 2:1 has been extensively explored via the study of fission isomers, for a long time the question on the existence of a hyperdeformed (i.e. axis ratio $3: 1$ ) third potential minimum remained unclear. In a series of measurements, employing light-particle induced direct reactions, in the isotopic chain of even-even uranium nuclei [13-15] (and recently in ${ }^{232} \mathrm{~Pa}$ for the first time also in an odd-A isotope [16]), we succeeded to establish the existence of a deep third potential minimum, in some cases comparably deep as the second minimum.

Since for hyperdeformed configurations the rather thin outer barrier results in fission lifetimes much shorter than typical lifetimes of collectively excited states in the excita- 


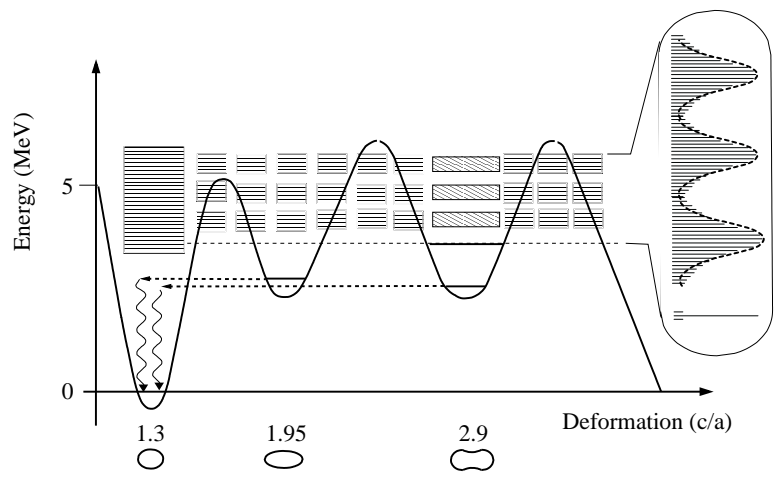

Fig. 2. Schematical description of the occurrence of transmission resonances in the prompt fission probability through a triplehumped fission barrier. An enhancement in the prompt fission probability can be observed at excitation energies, where Compound nuclear states populated in the first minimum energetically coincide with vibrational states in the third well.

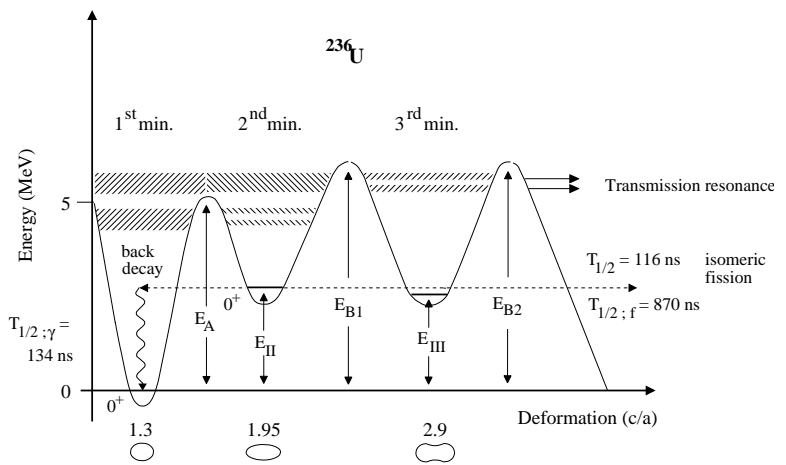

Fig. 3. The triple-humped potential-energy surface of ${ }^{236} \mathrm{U}$. Also damped class-I, class-II and class-III compound states are shown in the three minima. For strongly mixed class-I and class-II states, transmission resonances of class-III states can occur in fission.

tion energy region around ca. $1 \mathrm{MeV}$ below the barrier top, the method of transmission resonance spectroscopy was applied in all of these studies. Here the prompt fission cross section is measured, which due to subbarrier tunneling through the multiple-humped barrier exhibits resonant enhancements at excitation energies, where Compound nuclear states populated in the first minimum energetically coincide with vibrational states in the second or third potential well, respectively. For hyperdeformed transmission resonances (class-II states) to occur, it is necessary that the class-I and class-II compound states in the first and second minima are strongly mixed. Figure 2 illustrates the described occurrence of transmission resonances in the prompt fission cross section.

As a prototypic example of the potential energy landscape found in the chain of uranium isotopes, figure 3 displays the triple-humped fission barrier in ${ }^{236} \mathrm{U}$ as derived from our measurements [13].

Figure 4 shows an overview of the multiple-humped potential energy curves in even-even isotopes of thorium, uranium and plutonium nuclei. The curves have been obtained from theoretical calculations by Howard and Möller (for the first and second minima) [17], and by Cwiok et al. [18] for the hyperdeformed third minimum. Black dots denote experimental data for barrier height and potential minima, respectively. Open symbols indicate extrapolated expectations.

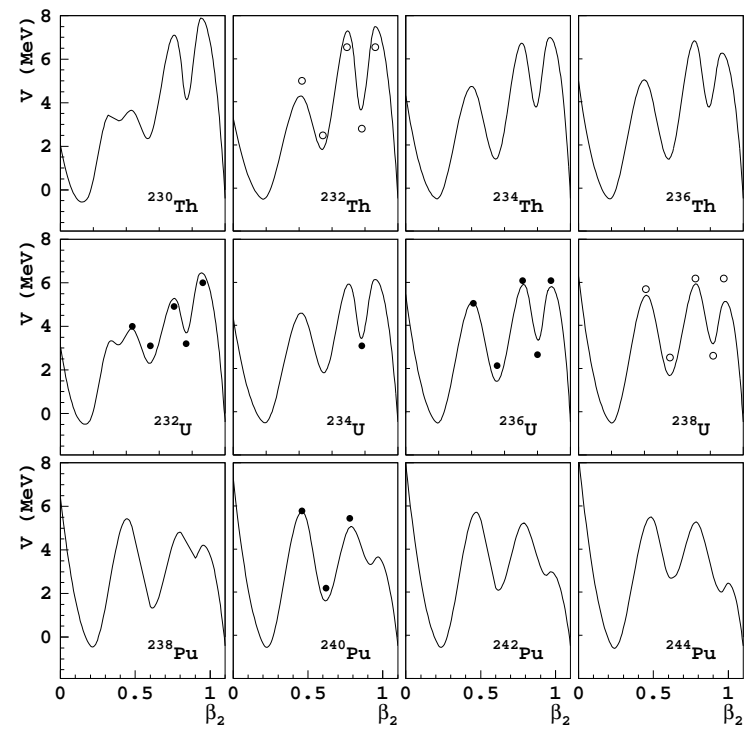

Fig. 4. Multiple-humped potential energy curves of even-even isotopes of thorium, uranium and plutonium, as obtained from theoretical calculations by Howard and Möller (for the first and second minima) [17] and by Cwiok et al. [18] for the hyperdeformed third minimum. Black dots denote experimental data for barrier height and potential minima, respectively $[13-15,19]$. Open symbols indicate our extrapolated expectations for ${ }^{232} \mathrm{Th}$ and ${ }^{238} \mathrm{U}$ as shown in figure 6 and figure 7.

Some trends can be inferred from this overview plot. The outer barrier height drops with increasing nuclear charge $\mathrm{Z}$, thus limiting the region where fission isomers can survive against prompt fission (i.e. the high- $Z$ shore of the 'island of fission isomers'). The inner barrier height drops with decreasing nuclear charge. For a long time, the socalled 'Thorium anomaly' was controversely discussed, which denoted a discrepancy between a theoretically predicted rather low inner fission barrier height (as also visible in figure 4) and the significantly (by about 2-3 MeV) contradicting experimental claim of a higher inner barrier. This discrepancy could only be resolved within our new paradigm of a deep third minimum, since the observation of a transmission resonance requires the fission barriers enclosing the respective super- or hyperdeformed state to be of about equal height. Within the new picture of a triplehumped fission barrier, this requirement can be met for the middle and outer barriers, while now the inner barrier height can be lowered as expected from theory. This also explains the lower- $Z$ shore of the 'island of fission isomers', where no fission isomers have been observed below $\mathrm{Z} \approx 90$. This can be explained by a lowering of the inner barrier height $\mathrm{E}_{A}$ with decreasing $\mathrm{Z}$, leading to a dominant fast $\gamma$ back-decay into the first minimum instead of isomeric fission. In addition of lowering of the inner barrier height was observed within the isotopic chain of uranium when going from heavier to lighter isotopes [14].

It should also be mentioned that recently the validity of the older theoretical calculations predicting the existence of a deep third minimum was questioned, concluding only in few cases like ${ }^{232} \mathrm{Th}$ the existence of a very shallow third well [20], while resulting in a drastic discrepancy with ex- 
perimental findings especially in the chain of uranium isotopes.

\subsection{Exploring the triple-humped fission barrier via photofission}

With the advent of next-generation $\gamma$-beam facilities like MeGaRay (Livermore, [5]) or ELI-Nuclear Physics (Bucharest/Romania, [9]), novel perspectives open up for photonuclear science in general and photofission studies of the multiple-humped fission barrier landscape in particular. So far studies in the second and third minimum of the potential energy surface had to rely on (light) particle-induced reactions in order to achieve a (statistical) population of states in these strongly deformed potential wells. With a typical population probability of $10^{-4}-10^{-5}$ for class-II and class-III states, very low isomeric fission rates of about 1 $\mathrm{s}^{-1}$ could be realized. Thus only strong transmission resonances with integrated cross sections of $\sigma \Gamma \sim 0.1 \mathrm{eVb}$ were accessible, hampered by the presence of a dominating background from prompt fission.

With the availability of an unprecedented quality of highlybrilliant, monochromatic $\gamma$ beams in the near future, photofission of actinides will experience a revival, reaching for so far inaccessible insights into structure and dynamics of the fission process from extremely deformed states. With the envisaged spectral photon density of $10^{4}-10^{6} \mathrm{\gamma} / \mathrm{eVs}$ and the low energy bandwidth of $\Delta \mathrm{E} / \mathrm{E} \leq 10-3$, a large increase of the isomeric fission yield of $10^{3}-10^{6} / \mathrm{s}$ can be expected. Due to the narrow energy bandwidth, background from prompt fission can be avoided almost completely, resulting in very clean spectra. Thus also weak fission resonances with $\Gamma \sigma \sim 0.1 \mathrm{eVb}$ will become accessible.

Most of our knowledge on photofission of actinides has been obtained in the past from experiments using bremsstrahlung beams with an effective energy bandwidth of $\Delta \mathrm{E}$ $\sim 200-300 \mathrm{keV}$. Here the photofission yield $Y_{f}$ has to be derived by folding the photofission cross section $\sigma_{f}$ with the exponentially decreasing bremsstrahlungs spectrum $N_{\text {brems }}$

$$
Y_{f}\left(J, K, E_{0}\right)=\int_{0}^{E o} \sigma_{f}\left(J, K, E_{0}\right) \cdot N_{\mathrm{brems}}\left(E_{0}, E_{\gamma}\right) \frac{d E_{\gamma}}{E_{\gamma}} .
$$

So far only very few photofission experiments in the past were conducted with $\gamma$ beams of narrow energy bandwidth, such as a study of the photofission cross section in ${ }^{232}$ Th using a tagged photon beam with an energy resolution of $14 \mathrm{keV}$ [21]. Fission resonances were observed at excitation energies of 5.5 and $5.6 \mathrm{MeV}$, respectively. However, no detailed nuclear structure information could be derived from this observation. Highest resolution so far was achieved by Zhang et al. [22], who exploited sharp ( $\mathrm{p}, \gamma)$ resonances in selected reactions on ${ }^{25} \mathrm{Mg},{ }^{42} \mathrm{Ca},{ }^{34} \mathrm{~S}$ and ${ }^{29} \mathrm{Si}$ targets to cover reaction-specific excitation energy regions between 5.86 and $6.18 \mathrm{MeV}$ with $\Delta \mathrm{E}=500 \mathrm{eV}$. However, for each $(\mathrm{p}, \gamma)$ reaction an energy region of only less than $10 \mathrm{keV}$ width could be studied. A rich structure of sharp resonances was observed in these narrow, yet rather arbitrarily chosen energy regions.

In the lower excitation energy range between $3.5 \mathrm{MeV}$ $-4.5 \mathrm{MeV}$, a characteristic kink in the fission yield distribution was observed, i.e. a deviation from the exponential
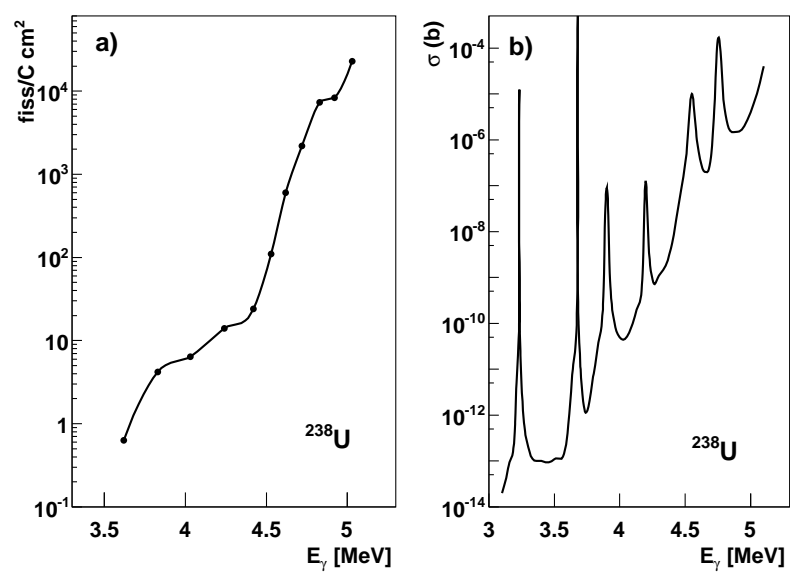

Fig. 5. a) Measured photofission yield and b) calculated total photofission cross section for dipole and quadrupole contributions, both displayed as a function of the maximum bremsstrahlung energy for photofission of ${ }^{238} \mathrm{U}$ [23].

decrease of the fission yield with decreasing excitation energy, the so-called 'isomeric shelf' $[23,24]$. It is caused by a competition of prompt and delayed (isomeric) fission in this energy region.

Figure 5 displays the isomeric shelf as obtained in theoretical studies [23] for ${ }^{238} \mathrm{U}$. According to [23], the isomeric shelf consists of contributions from $2^{+}(\mathrm{K}=0)$ quadrupole and $1^{-}(\mathrm{K}=0)$ dipole resonances as gateway states to the second minimum. Here the $1^{-}$contribution dominates around $4 \mathrm{MeV}$. The left panel of figure 5 shows the fission yield as obtained from bremsstrahlung experiments with $\Delta \mathrm{E} \sim 200 \mathrm{keV}$. However, as outlined by [23], the broad structure of the isomeric shelf is predicted to be intrinsically composed of a multitude of individual sharp resonances, which so far could not be resolved.

All these findings clearly indicate the presence of sharp fission resonances throughout the energy range around the fission barrier top down to the region of the isomeric shelf, calling for a consistent investigation over the full energy range with highest resolution. A wealth of new insights into the structure of the actinide potential barrier landscape awaits disclosure, particularly in the extremely deformed second and third potential minima. Now, with the novel upcoming $\gamma$ beams potentially reaching an energy bandwidth (after monochromatization) of $10^{-6}$, the ultimate photon beam energy resolution may be ideally matched to the intrinsic linewidth of individual nuclear resonances, given by the thermal Doppler broadening according to

$$
\Delta E / E \sim 10^{-6} \cdot \sqrt{k T /(25 m e V) \cdot(240 / A)} .
$$

Thus for $\mathrm{A}=240$ an intrinsic linewidth of $\Delta \mathrm{E} / \mathrm{E} \sim 10^{-6}$ is expected, corresponding to $\delta \mathrm{E}=5 \mathrm{eV}$ at $5 \mathrm{MeV}$. As discussed before, such an energy bandwidth may be experimentally accessible in the future by combining the excellent properties of next-generation $\gamma$ beams with the newly developed refractive $\gamma$ focusing optics. If so, resolving the underlying fission resonance structure of the isomeric shelf as well as the full picture of the fission resonance structure at higher excitation energies will come into experimental reach for the first time.

Particularly interesting candidates for these studies at ELI-NP are ${ }^{232} \mathrm{Th}$ and ${ }^{238} \mathrm{U}$, respectively. While so far in 


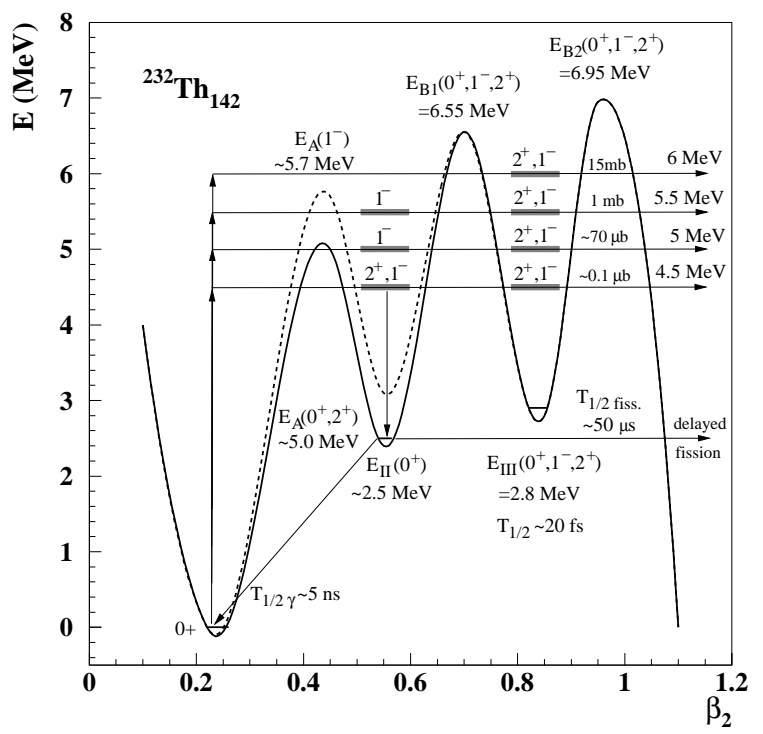

Fig. 6. Schematical view of our expectation for the features of the triple-humped fission barrier in ${ }^{232} \mathrm{Th}$, based on the assumption of a deep third minimum as already established in the neighbouring chain of uranium isotopes. The dashed curve represents the inner barrier height for dipole contributions, thus illustrating representing the spin-dependency of the barrier at the first saddle point [25].

${ }^{232} \mathrm{Th}$ not even the existence of a fission isomer in the second potential minimum could be directly observed, Zhang et al. [22] concluded via level density arguments from their photofission data the ground-state excitation energy of the second potential minimum as $2.8 \mathrm{MeV}$. However, at the time only a very shallow third minimum was assumed in their analysis. With our refined present knowledge on the existence of deep third potential wells in the actinides, we are tempted to interpret the result of Ref. [22] not as the ground state of the second, but rather of the (hyperdeformed) third potential minimum. Figure 6 gives our tentative picture on the triple-humped fission barrier in ${ }^{232} \mathrm{Th}$, together with indications of expected dipole and quadrupole fission resonances. This picture could be tested with a monochromatic $\gamma$ beam at ELI-NP.

A second promising case to be studied is ${ }^{238} \mathrm{U}$, where so far only one experiment exploiting the $(t, p)$ reaction is reported [26]. Strong resonances were observed with moderate energy resolution (ca. $50 \mathrm{keV}$ ), however, no highresolution photofission experiment could since then be performed. Putting together all information available so far on this isotope, figure 7 displays our expectation for the features of the fission barrier in ${ }^{238} \mathrm{U}$, where we assume, besides the properties of the well-known second minimum at $\mathrm{E}^{*}=2.56 \mathrm{MeV}$, a third minimum of about equal depth. Fission resonances should appear in the prompt fission cross section at about 5.6 MeV, originating from the second potential minimum, while for lower excitation energies resonances from the third potential well are predicted to appear around 5.15 MeV and 4.5 MeV, respectively.

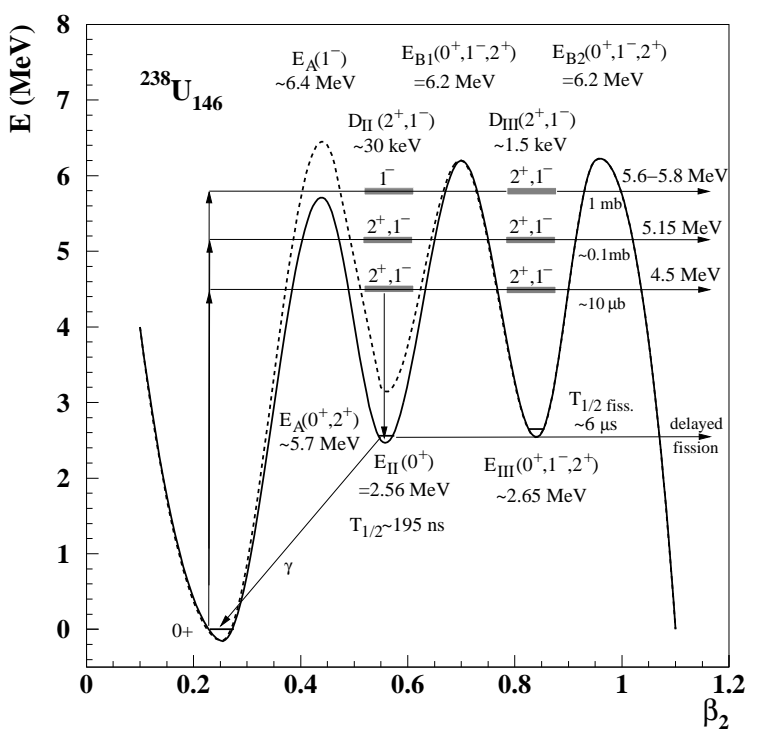

Fig. 7. Schematical view of our expectation for the features of the triple-humped fission barrier in ${ }^{238} \mathrm{Th}$, assuming a deep third minimum of about the same depth as the well-established second minimum. The dashed curve represents the inner barrier height for dipole contributions, thus illustrating the spin-dependency of the barrier at the first saddle point [25].

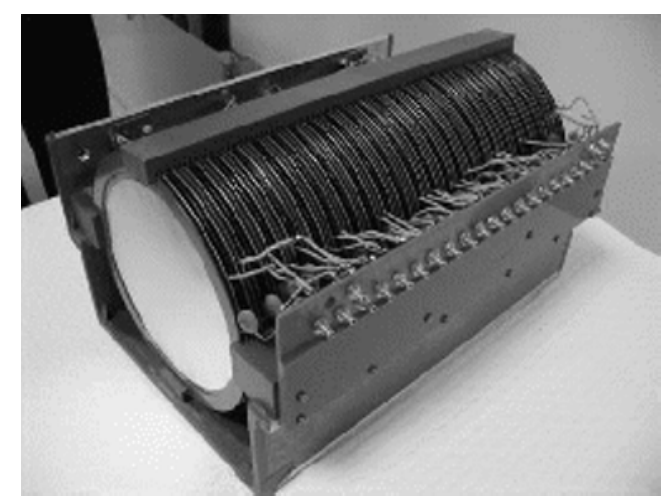

Fig. 8. Parallel plate detector array for the coincident detection of fission fragments of ${ }^{238} \mathrm{U}$, consisting of a stack of $23{ }^{238} \mathrm{UO}_{2}$ targets mounted inbetween parallel plate detector electrodes [27].

\subsection{Exploratory photofission experiment}

In order to prepare for photofission experiments at the nextgeneration $\gamma$-beam facilities like ELI-NP, an exploratory experiment has been performed at the $\mathrm{HI} \gamma \mathrm{S}$ facility (Duke University), studying photofission in ${ }^{238} \mathrm{U}$ between $\mathrm{E}^{*}=$ 4.8 - 6.2 MeV. A multi-target PPAC coincidence array [27], comprised of 23 large-area ${ }^{238} \mathrm{UO}_{2}$ targets (diameter 6.5 $\mathrm{cm}$, thickness $2 \mathrm{mg} / \mathrm{cm}^{2}$ ), was used to measure the photofission cross section from an incident photon beam with typically $\Delta \mathrm{E} / \mathrm{E} \sim 160-200 \mathrm{keV}$ and a flux of $2-4 \cdot 10^{7} \gamma / \mathrm{s}$ on target. The detector array is shown in figure 8 .

The measured fission cross section can be seen in figure 9 [28]. Compared to the so far only reported crosssection measurement [29], our experiment extended the covered range of excitation energies by about an order of magnitude to lower cross sections. 


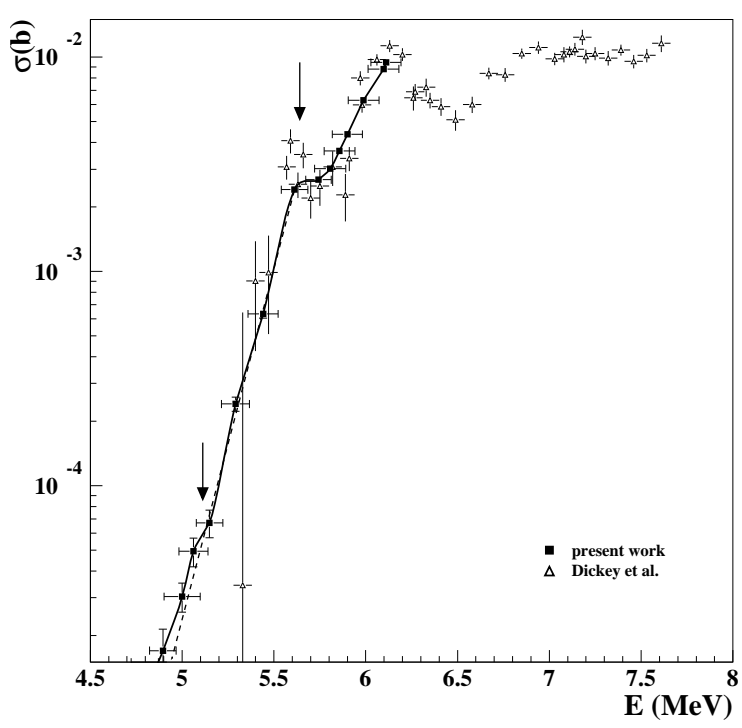

Fig. 9. Photofission cross section for ${ }^{238} U$ as measured in our exploratory experiment at the $\mathrm{HI} \gamma \mathrm{S}$ facility (full symbols) compared to previous data from Ref. [29] (open symbols). The two arrows mark the positions for expected fission resonances from the second and third potential minimum, respectively. The dashed line marks the exponential drop of the fission probability below the barrier top.

The spectrum contains dipole $\left(1^{-}\right)$and quadrupole $\left(2^{+}\right)$ contributions. The previously known resonance at $5.6 \mathrm{MeV}$, originating from the second potential minimum, where dipole and quadrupole components belong to different, wellseparated bands, could be confirmed as a shoulder in our data. The expected hyperdeformed resonance at around 5.15 $\mathrm{MeV}$, originating from one octupole-deformed band, is hardly visible as slight deviation from the exponential drop of the prompt fission cross section (indicated by the dashed line).

Clearly much better energy resolution of the $\gamma$ beam (as envisaged for ELI-NP) is required for any clear evidence in this deep sub-barrier energy region. With the energy resolution envisaged for ELI-NP of $\Delta \mathrm{E} / \mathrm{E}$ better than $10^{-3}$, and thus by more than an order of magnitude better than presently reached at $\mathrm{HI} \gamma \mathrm{S}$ for $5 \mathrm{MeV}$, together with the expected drastic improvement of the $\gamma$-beam brilliance by about 5 orders of magnitude, the intrinsically narrow low-energy fission resonances like the one at $5.1 \mathrm{MeV}$ can be expected to be clearly visible.

\subsection{Developments for novel $\gamma$ beams}

While for the above described exploratory experiment an existing detector system was used, which was developed for bremsstrahlung photon beams with rather large beam diameters of a few centimeters, the novel $\gamma$ beams from Compton backscattering facilities will exhibit very much reduced beam diameters. Therefore much smaller targets and much more compact detector systems can be used, adapted for the small beam-spot size (well below a millimeter diameter). This will enable to reduce the amount of target material needed, allowing the use of expensive

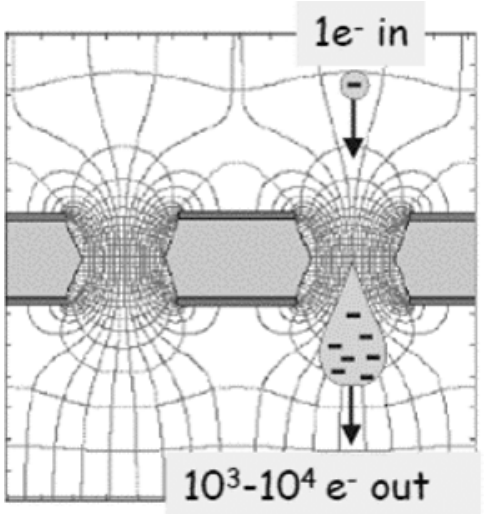

Fig. 10. Principle of electron amplification in the strong electric field of a membrane pore (hole) in a (Thick) GEM detector [30].
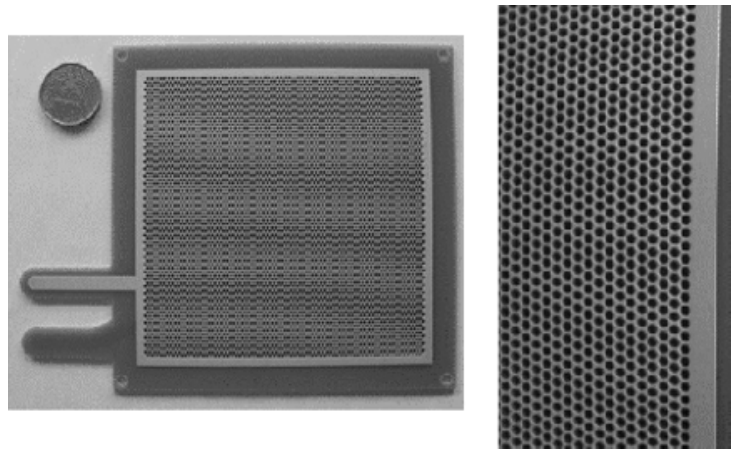

Fig. 11. Left: Thick-GEM board with a thickness of $1.6 \mathrm{~mm}, 1$ $\mathrm{mm}$ hole diameter and a pitch size of $1.5 \mathrm{~mm}$. Right: close view to the etched holes.

rare isotopes together with a drastic reduction of the target radioactivity of e.g. actinide targets.

Moreover, also the detector systems will be adapted to these new conditions. Much more compact gas detector arrays will be developed for fission yield measurements, resulting also in an improved time resolution for coincidence measurements. Position-sensitive detectors will allow to measure the fission fragment angular distribution, allowing to extract spins and $\mathrm{K}$ values of fission resonances, especially for $1^{-}$and $2^{+}$. We already started the development of an efficient multi-target, 2D position sensitive detector array, based on the Thick GEM (Gas Electron Multiplier) technology [30]. Figure 10 illustrates the principle of GEM detectors.

A membrane is placed into a gas volume and high voltage is applied between its two surfaces. The resulting strong electric field in the pores (typically $50 \mu$ m diameter) leads to strong amplification of electrons from ionization processes of incident particles or radiation [31]. When used for X-ray or single-photon counting, typically several GEM amplification stages (each with a typical amplification factor of $10^{4}$ ) are used. In case of the Thick GEM technique, the fine membrane is replaced by a printed circuit board with etched holes (typical hole diameter $1 \mathrm{~mm}$, pitch size $1.5 \mathrm{~mm}$ ). Figure 11 shows on the left side a typical Thick GEM cathode board as used for our detector developments, the right part shows a close view to the etched hole structure.

Since fission fragments will deposit a large amount of energy and thus cause massive ionization in the gas vol- 
ume, a single-stage detector is sufficient to generate large signals at the $2 \mathrm{D}$-segmented anode providing the signal readout.

\subsection{Conclusion: Perspectives for photofission with monochromatic $\gamma$ beams}

With the upcoming highly briliant, monochromatic $\gamma$ beams of ELI-NP or MEGa-Ray, unprecedented beam properties will be available for photonuclear science in general and photofission studies in particular. The very low energy bandwidth, ultimately envisaged to reach values of $\Delta \mathrm{E} / \mathrm{E} \approx 10^{-5}$ $10^{-6}$ (after efficient monochromatization with novel focusing optics) and thus close to the inherent limit by thermal Doppler broadening, will for the first time allow for a stateselective $\gamma$ spectroscopy, e.g. enabling to resolve the wellknown 'isomeric shelf' in the photofission cross section into its predicted underlying individual resonances. Since incident photons can be tuned to excite only selected nuclear resonances, very clean spectra will result from a drastic reduction of background from atomic processes like Compton scattering or pair creation.

A wealth of new opportunities for mapping the multiplehumped potential energy landscape of actinides and for investigating nuclei with exotic shapes and/or large deformations (fission isomers, hyperdeformed resonances) will emerge.

It will become possible to study the $\gamma$ decay within the second minimum (e.g. in Th isotopes) and the subsequent back-decay to the first minimum. Hence excitation energies of strongly deformed resonances and isomeric states can be identified with unprecedented energy resolution. Mul ti-phonon $\beta$-vibrational resonances can be mapped, thus allowing to test the harmonicity of the potential barrier from low-lying collective states to multi-phonon excitations close to the barrier top. Hereby speculations on a potential tower-like shape of the fission barrier in specific isotopes can be tested.

Besides access to these basic properties, there exists also a strong application potential. Specific resonances in the (hyper-deformed) third minimum may exhibit a strongly enhanced E1 strength, e.g. via the low-energy component of the Giant Dipole Resonance. Compared to a typical E1 strength of $10^{-3} \mathrm{WU}$ in the normal-deformed first minimum, an enhancement by up to three orders of magnitude may be found. With such an increased E1 absorption as doorway state to fission, selective and efficient transmutation of minor actinides could be realized and become an important contribution to the widely discussed issue of nuclear waste management.

Finally we conclude that highly brilliant, monochromatic $\gamma$ beams thus have the potential to lift photonuclear science into a new era of 'nuclear photonics', doing for nuclear isotopes what the laser decades ago did for atomic physics.

This work was supported by the DFG Cluster of Excellence 'Origin and Structure of the Universe'.

\section{References}

1. U. Kneissl, et al., Prog. Part. Nucl. Phys. 37, 349 (1996); U. Kneissl, N. Pietralla, A. Zilges, J. Phys. G:
Nucl. Part. Phys. 32, R217 (2006)

2. U. Kneissl, et al., Fortschritte der Physik 30, 311 (1982)

3. L. Schnorrenberger, et al., Jour. Phys. Conf. Ser. 202, 012027 (2010)

4. H.R. Weller, et al., Prog. Nucl. Part. Phys. 62, 257 (2008)

5. C. Barty (LLNL), ELI-NP-meeting, http://www.elinp.rp/gamma-beam-meeting-august-presentation.php

6. S.G. Anderson, et al., Appl. Phys. B 78, 891 (2004)

7. https://newsline.llnl.gov/articles/2008/apr/04.04.08_TREX.php

8. D. Belic et al., Nucl. Instr. Meth. A 463, 26 (2001)

9. http://www.eli-np.ro/; http:/www.eli-np.ro/excecutive committee-meeting-april12-13.php (2010).

10. D. Habs, et al., Phys. Rev. Lett. 108, 184802 (2012)

11. P.G. Thirolf and D. Habs, Prog. Part. Nucl. Phys. 49, 325 (2002)

12. A. Krasznahorkay, Handbook of Nuclear Chemistry, 1 (Springer Verlag, Hamburg 2011)

13. M. Csatlos, et al., Phys. Lett. B 615, 175 (2005)

14. L. Csige, et al., Phys. Rev. C 80, 011301 (2009)

15. A. Krasznahorkay, et al., Phys. Lett. B 461, 15 (1999)

16. L. Csige, et al., Phys. Rev. C 85, 054306 (2012)

17. W.M. Howard and P. Möller, At. Data \& Nucl. Data Tables 25, 219 (1980)

18. S. Cwiok, et al., Phys. Lett. B 322, 304 (1994)

19. M. Hunyadi, et al., Phys. Lett. B 505, 27 (2001)

20. M. Kowal and J. Skalski, Phys. Rev. C 85, 061302 (2012)

21. J.W. Knowles, et al., Phys. Lett. B 116, 315 (1982)

22. H.X. Zhang, et al., Phys. Rev. Let. 53, 34 (1984)

23. G. Bellia, et al., Z. Phys. A 314, 43 (1983)

24. C.D. Bowman, Phys. Rev. C 12, 856 (1975); C.D. Bowman, et al., Phys. Rev. C 12, 863 (1975)

25. R. Vandenbosch, Phys. Lett. B 45, 207 (1973)

26. B.B. Back, et al., Phys. Rev. C 9, 9 (1974)

27. J. Drexler, et al., Nucl. Phys. A 411, 17 (1983); J. Drexler, et al., Nucl. Instr. Meth. 220, 409 (1984)

28. L. Csige, et al., (to be published)

29. P.A. Dickey and P. Axel, Phys. Rev. Lett. 35, 501 (1975)

30. R. Chechik, et al., Nucl. Instr. Meth. A 535, 303 (2004)

31. F. Sauli, et al., Nucl. Instr. Meth. A 386, 531 (1997) 